\title{
Elastic electron scattering from methane at high momentum transfer
}

\author{
M Vos ${ }^{1}$, M R Went ${ }^{1}$, G Cooper ${ }^{2}$ and C A Chatzidimitriou-Dreismann ${ }^{3}$ \\ ${ }^{1}$ Atomic and Molecular Physics Laboratories, Research School of Physical Sciences and Engineering, \\ Australian National University, Canberra ACT 0200, Australia \\ ${ }^{2}$ Department of Chemistry, McMaster University, Hamilton, On. L8S 4M1, Canada \\ ${ }^{3}$ Institute of Chemistry, Technical University Berlin, D-10623 Berlin, Germany \\ E-mail: Maarten.Vos@anu.edu.au
}

Received 17 April 2008, in final form 8 May 2008

Published 23 June 2008

Online at stacks.iop.org/JPhysB/41/135204

\begin{abstract}
We describe elastic electron scattering data at high momentum transfer (between $\approx 20$ and $\approx 40 \mathrm{au}$ ) from methane and $\mathrm{Xe}$. Under these conditions there is a significant recoil energy transferred to the target and electrons scattered elastically from methane are separated into two peaks: one due to electrons scattered from carbon, and one due to electrons scattered from hydrogen. The separation of these peaks is within a few per cent identical to what is expected for scattering from isolated $\mathrm{C}$ and $\mathrm{H}$ atoms. The peak due to electrons scattered from $\mathrm{C}$, is again shifted compared to the peak of electrons scattered from $\mathrm{Xe}$. The $\mathrm{Xe}, \mathrm{C}$ and $\mathrm{H}$ peaks all have clearly different widths. The $\mathrm{C}$ and $\mathrm{H}$ peak areas are compared. Their relative intensity shows no substantial deviation $(<10 \%)$ from what is expected based on either simple Rutherford cross sections, or state-of-the-art elastic scattering calculations. The latter observation is in strong contrast to electron scattering results from a gaseous equimolar $\mathrm{H}_{2}-\mathrm{D}_{2}$ mixture and from electron and neutron scattering results from polymers at similar momentum transfer.
\end{abstract}

(Some figures in this article are in colour only in the electronic version)

\section{Introduction}

In elastic electron scattering experiments one tends to consider the energy of the scattered electron unchanged. However momentum conservation dictates that, if the momentum of the scattered electron changes by $\boldsymbol{q}$, then the momentum of the scatterer will change by $\boldsymbol{- q}$ in these collisions. Thus, implicitly one makes the assumption that the mass of the scatterer (a nucleus or molecule of mass $M$ ) is so large that the energy transfer from an electron to a nucleus (corresponding to $q^{2} / 2 M$ ) can be neglected. This energy transfer is largest for scattering over $180^{\circ}$ and is then (for scattering from a stationary nucleus at non-relativistic electron energies) simply $\frac{4 m}{M} E_{0}$, with $E_{0}$ the energy of the impinging electron. At $2 \mathrm{keV}$, for example, this energy transfer is $\approx 4.36 \mathrm{eV}$ for scattering from a hydrogen atom. Such energies are easily resolved in a modern electron spectrometer. Thus, this makes a new class of experiments possible, and requires us to reconsider some aspects of our basic understanding of 'elastic scattering'. For example, if we scatter from a hydrogen molecule, should we consider the mass of a hydrogen atom or the hydrogen molecule when calculating the energy transfer? Can we consider the target as stationary, or do their translational and vibrational properties come into play? Here we describe an experiment of electron scattering from methane, and investigate if the observed phenomena can be explained using known properties of methane.

The first observation of energy transfer to a nucleus due to elastic scattering can be found in the work of Boersch and co-workers (Boersch et al 1967). They investigated whether an electron scattered from a target interacts with the whole target, or with a single nucleus, and observed an energy shift $(\approx 2.8 \mathrm{eV})$ for $30 \mathrm{keV}$ electrons scattering from a carbon film over $90^{\circ}$. This shift was consistent with momentum transfer to a single atom. They also observed a remarkably large width of the elastic peak, which they attributed to sample heating due to their relatively intense electron beam. More recently these electron scattering experiments from surfaces were done at lower energies and shifts of the elastic peak of 
several elements were resolved (Varga et al 2001, Werner et al 2001). The signal of hydrogen in polymers and hydrogenated amorphous carbon was separated from the carbon elastic peak (Vos 2002, Yubero et al 2005, Varga et al 2006).

Independent of this, neutron scattering of epi-thermal neutrons was developed at spallation sources. These experiments have comparable momentum transfer to $\mathrm{keV}$ large-angle electron scattering experiments. It was suggested early that the energy distribution of a scattered neutron would reflect the momentum of the scattering atom (Hohenberg and Platzman 1966) in the same way that a scattered photon reflects the momentum of the scattering electron in a Compton experiment (Cooper et al 2004). Hence the term neutron Compton scattering was coined (Watson 1996) and this technique provides very direct information on momentum distributions of nuclei in solids.

It was later established that neutron scattering and electron scattering at the same momentum transfer are directly comparable and contain the same signature of the nuclear motion (Vos et al 2004), and that both techniques have problems reconciling the intensity of the hydrogen peak and carbon peak in polymers (Chatzidimitriou-Dreismann et al 2003, Vos et al 2004). These experiments are part of a larger body of neutron data where the observed intensity of the hydrogen signal seems at odds with the expected intensity based on the well-known cross section (see e.g. Chatzidimitriou-Dreismann et al 1997 or AbdulRedah and Chatzidimitriou-Dreismann 2002), deviations that were interpreted as a sign of the quantum nature (in particular attosecond entanglement) of protons in matter. The interpretation of these measurements remains however highly controversial (Karlsson and Lovesey 2000, Cowley 2003, Karlsson 2003, Reiter and Platzman 2005, Gidopoulos 2005, Chatzidimitriou-Dreismann 2005, ChatzidimitriouDreismann and Stenholm 2007) and a consensus on the cause of the anomalous hydrogen intensity has not been reached. Thus our present understanding of high momentum-transfer collisions (both in neutrons and in electron scattering) is far from complete.

In order to advance our understanding it is thus highly desirable to study simpler systems, in particular gas-phase scattering experiments. Here we think electron scattering can contribute greatly to the discussion as these targets are, due to their low density, not so easily accessible by neutron scattering. The much stronger interaction of an electron (compared to a neutron) with the target can now be used to our advantage. In electron scattering experiments from solids, the stronger interaction leads to multiple scattering (and possibly radiation damage), complicating the interpretation of the data. In a gas-phase experiment the target density is such that for electrons the probability of multiple scattering is small, an assumption that can be easily tested by changing the gas pressure. At the same time count rates, especially when employing modern two-dimensional detection techniques, are still reasonable. A beginning was made in the work by Cooper et al (2007). However, a full separation of the $\mathrm{H}$ and $\mathrm{C}$ derived peaks was not obtained in that work. Hence the area ratio obtained depends, as we will show, somewhat on the fitting procedure followed. In the present paper we increase considerably the range of momentum transfer studied, enhancing the carbon-hydrogen separation by up to a factor of 4 , and at the same time we have increased the energy resolution somewhat. This makes extracting data from the measurement much more straightforward and a quite stringent comparison of the measured intensity with the expected one becomes possible. This is the subject of this paper.

\section{Theory}

For high momentum transfer experiments, when the recoil energy $q^{2} / 2 M_{H}$ is larger than the experimental energy resolution, the signal of elastically scattered electrons from methane consists of two peaks: a main peak and a much less intense peak at somewhat lower kinetic energy. This is already clear from the work of Cooper et al (2007). The splitting of these two peaks is consistent with a reduction in the electron's kinetic energy by an amount $q^{2} / M_{H, C}$ with $M_{H}$ and $M_{C}$ the mass of a $\mathrm{H}$ and $\mathrm{C}$ atom, respectively. Thus it appears as if we scatter from isolated atoms, rather than a molecule, and this observation is consistent with the work of Boersch et al (1967) for scattering of energetic electrons over large angles from graphite.

Theoretically, collisions at similar momentum transfer have been studied extensively by neutron scattering (see e.g. Watson 1996 for a review), and we think that the main conclusions apply to electron scattering as well. In particular, the energy after elastic scattering is that expected for scattering from a single atom within the molecule, rather than the molecule as a whole if $|q||r| \gg 1$, with $|r|$ the interatomic separation. This is referred to as the incoherent approximation. Under these conditions scattering waves emanating from different atoms have relative phases that change so rapidly with $r$ (due to vibrations, or even the zero-point motion) that diffraction effects average out to 0 . A second important issue, as established in the neutron scattering theory, is that of the collision time. The collision occurs in such a short time (given in atomic units approximately by $\tau=\frac{M}{q}\left\langle p_{q}^{2}\right\rangle$, with $p_{q}$ the momentum component of the scatterer along the momentum transfer direction), that the scattering atom has no time to move, and hence is not affected by interactions with neighbouring atoms. Hence the atom acts, as far as the collision is concerned, as a free atom. This is referred to as the plane-wave-impulse approximation. Under these conditions one can interpret the observed energy distribution of the scattered electron as a Compton profile of the momentum distribution of the scattering atom, and the transferred energy can be calculated assuming scattering from a free atom with momentum $p$ :

$$
E_{r}=\frac{(\boldsymbol{p}+\boldsymbol{q})^{2}}{2 M}-\frac{p^{2}}{2 M}=\frac{q^{2}}{2 M}+\frac{\boldsymbol{p} \cdot \boldsymbol{q}}{M} .
$$

Thus the observed energy distribution reflects directly the probability that the atom had, before the collision, a certain momentum component along the momentum-transfer direction. This momentum distribution is at high temperatures that of a classical gas, but at low temperatures determined 
Table 1. The differential cross sections at angles and energies used in this paper for electrons scattering from $\mathrm{C}$ and $\mathrm{H}$. The Rutherford cross section assumes scattering from a bare nucleus. The differential cross sections obtained using the ELSEPA package (Salvat 2005) without and with absorption are also given.

\begin{tabular}{|c|c|c|c|c|c|c|c|c|}
\hline \multirow[b]{2}{*}{$\begin{array}{l}E_{0} \\
(\mathrm{keV})\end{array}$} & \multirow[b]{2}{*}{$\begin{array}{l}\theta \\
\left({ }^{\circ}\right)\end{array}$} & \multirow[b]{2}{*}{$\begin{array}{l}q \\
\text { (au) }\end{array}$} & \multicolumn{2}{|c|}{ Rutherford } & \multicolumn{2}{|c|}{ ELSEPA no abs. } & \multicolumn{2}{|c|}{ ELSEPA incl. abs. } \\
\hline & & & $\begin{array}{l}\frac{\mathrm{d} \sigma}{\mathrm{d} \Omega} \mathrm{C} \\
(\mathrm{au})\end{array}$ & $\begin{array}{l}\frac{\mathrm{d} \sigma}{\mathrm{d} \Omega} \mathrm{H} \\
(\mathrm{au})\end{array}$ & $\begin{array}{l}\frac{\mathrm{d} \sigma}{\mathrm{d} \Omega} \mathrm{C} \\
(\mathrm{au})\end{array}$ & $\begin{array}{l}\frac{\mathrm{d} \sigma}{\mathrm{d} \Omega} \mathrm{H} \\
(\mathrm{au})\end{array}$ & $\begin{array}{l}\frac{\mathrm{d} \sigma}{\mathrm{d} \Omega} \mathrm{C} \\
(\mathrm{au})\end{array}$ & $\begin{array}{l}\frac{\mathrm{d} \sigma}{\mathrm{d} \Omega} \mathrm{H} \\
(\mathrm{au})\end{array}$ \\
\hline 1.55 & 90 & 15.1 & $2.78 \times 10^{-3}$ & $7.72 \times 10^{-5}$ & $3.02 \times 10^{-3}$ & $7.84 \times 10^{-5}$ & $2.61 \times 10^{-3}$ & $7.46 \times 10^{-5}$ \\
\hline 2.00 & 90 & 17.1 & $1.67 \times 10^{-3}$ & $4.65 \times 10^{-5}$ & $1.81 \times 10^{-3}$ & $4.69 \times 10^{-5}$ & $1.60 \times 10^{-3}$ & $4.52 \times 10^{-5}$ \\
\hline 2.65 & 90 & 19.7 & $9.57 \times 10^{-4}$ & $2.66 \times 10^{-5}$ & $1.03 \times 10^{-3}$ & $2.66 \times 10^{-5}$ & $9.26 \times 10^{-4}$ & $2.59 \times 10^{-5}$ \\
\hline 4.00 & 90 & 24.3 & $4.20 \times 10^{-4}$ & $1.17 \times 10^{-5}$ & $4.46 \times 10^{-4}$ & $1.17 \times 10^{-5}$ & $4.12 \times 10^{-4}$ & $1.14 \times 10^{-5}$ \\
\hline 6.00 & 90 & 29.8 & $1.87 \times 10^{-4}$ & $5.20 \times 10^{-6}$ & $1.96 \times 10^{-4}$ & $5.17 \times 10^{-6}$ & $1.85 \times 10^{-4}$ & $5.10 \times 10^{-6}$ \\
\hline 8.00 & 90 & 34.4 & $1.06 \times 10^{-4}$ & $2.94 \times 10^{-6}$ & $1.09 \times 10^{-4}$ & $2.90 \times 10^{-6}$ & $1.04 \times 10^{-4}$ & $2.88 \times 10^{-6}$ \\
\hline 1.55 & 135 & 19.7 & $9.55 \times 10^{-4}$ & $2.65 \times 10^{-5}$ & $1.14 \times 10^{-3}$ & $2.66 \times 10^{-5}$ & $1.02 \times 10^{-3}$ & $2.57 \times 10^{-5}$ \\
\hline 2.00 & 135 & 22.4 & $5.74 \times 10^{-4}$ & $1.59 \times 10^{-5}$ & $6.65 \times 10^{-4}$ & $1.61 \times 10^{-5}$ & $6.00 \times 10^{-4}$ & $1.55 \times 10^{-5}$ \\
\hline 4.00 & 135 & 31.7 & $1.44 \times 10^{-4}$ & $4.00 \times 10^{-6}$ & $1.55 \times 10^{-4}$ & $3.95 \times 10^{-6}$ & $1.44 \times 10^{-4}$ & $3.87 \times 10^{-6}$ \\
\hline 6.00 & 135 & 38.9 & $6.42 \times 10^{-5}$ & $1.78 \times 10^{-6}$ & $6.72 \times 10^{-5}$ & $1.76 \times 10^{-6}$ & $6.35 \times 10^{-5}$ & $1.74 \times 10^{-6}$ \\
\hline 2.25 & 100 & 19.7 & $9.60 \times 10^{-4}$ & $2.67 \times 10^{-5}$ & $1.06 \times 10^{-3}$ & $2.69 \times 10^{-5}$ & $9.47 \times 10^{-4}$ & $2.60 \times 10^{-5}$ \\
\hline
\end{tabular}

by properties of the scattering system (see e.g. Fielding et al 2000). At $0 \mathrm{~K}$ the measured distribution reflects the zero-point motion. Thus although the collision appears to be a collision between free particles, it allows for measurement of the properties of atoms bound to a larger system. The mentioned approximations are expected to become more precise with increasing magnitude of $\boldsymbol{q}$. Here we shall generally assume that these approximations are valid.

If one resolves the elastic peak of methane in two components then one can compare the intensity of both peaks. What should one expect for the ratio? Classically these largeangle high-momentum-transfer collisions would be described by trajectories that approach the nucleus very closely; for example, the minimum distance of a $2 \mathrm{keV}$ electron that is deflected from carbon nucleus over $180^{\circ}$ is only $0.08 \mathrm{au}$. If this distance is much smaller than the size of the electron cloud then one could forget in first approximation about the effect of screening of the nuclear charge on the interaction with this electron, and then the Rutherford cross section would apply. It scales as $Z^{2}$, and in this approximation the $\mathrm{C}$ cross section would be 36 times the $\mathrm{H}$ elastic scattering cross section.

A somewhat less crude approximation would be to assume that scattering from $\mathrm{CH}_{4}$ happens, for all intents and purposes, near either the $\mathrm{C}$ or an $\mathrm{H}$ nucleus and that the electron density near the nucleus is not affected in a major way by the chemical bond. Thus screening effects, due to electrons near the nucleus, can be accounted for by calculating the differential elastic scattering cross section for $\mathrm{H}$ and $\mathrm{C}$ atoms. For this we used the ELSEPA package developed by Salvat et al (2005), and results are given, together with the Rutherford cross section in table 1. One assumes that the elastic differential cross section can be calculated from the potential of an $\mathrm{H}$ or $\mathrm{C}$ atom. Especially for hydrogen the difference between the Rutherford cross section and the ELSEPA derived cross section is small. These calculations are for potential scattering. There is no energy transfer in these calculations.

ELSEPA has the option to include the effect of the absorption potential, and calculations were done with and without absorption. Absorption (describing the reduction in the elastic peak due to inelastic processes that may occur while

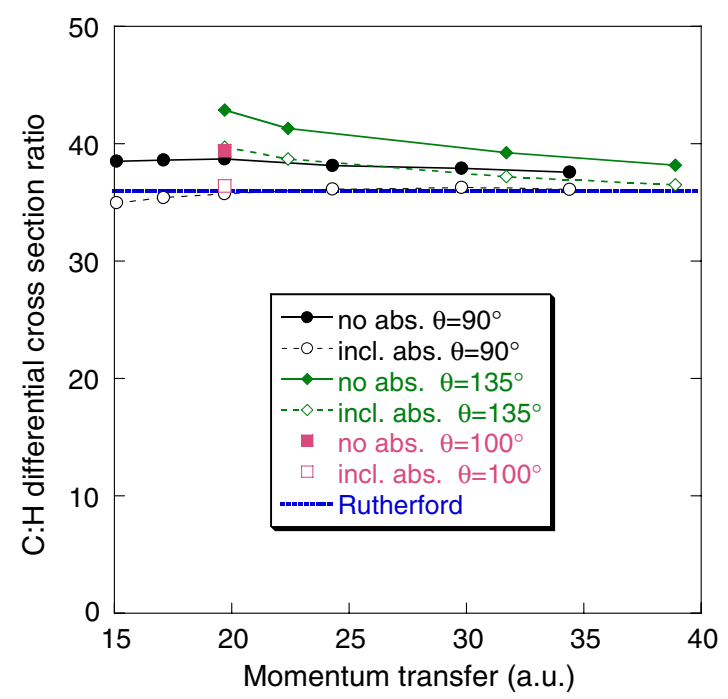

Figure 1. The ratio of the $\mathrm{C}: \mathrm{H}$ cross section as calculated using the ELSEPA package (with and without absorption) (Salvat 2005) and by the Rutherford formula. The ratio is, using any of these approximations, within $10 \%$ of $36: 1$ (except for the $135^{\circ}$ calculation at 1.55 and $2 \mathrm{keV}$ where the calculated ratio without absorption deviates by $\approx 15 \%$ ).

the electron is deflected) is the least well-tested contribution to the elastic cross section at these high energies, and the program uses a LDA-type approach with a not very rigorously-defined parameter for the absorption potential strength. We used the recommended value of 2.0 for this parameter, which gives results that are known to be in reasonable agreement with experimentally obtained differential cross sections (Salvat 2003).

Of more importance to this experiment is the ratio of the $\mathrm{C}$ and $\mathrm{H}$ differential cross section. This is plotted in figure 1 for the values of interest to this paper as a function of the momentum transfer. If the first Born approximation was a valid description of the experiment then this ratio (and even the cross sections themselves) should be dependent only on the momentum transfer. Clearly the calculated cross section ratio at the same momentum transfer depends slightly on the 


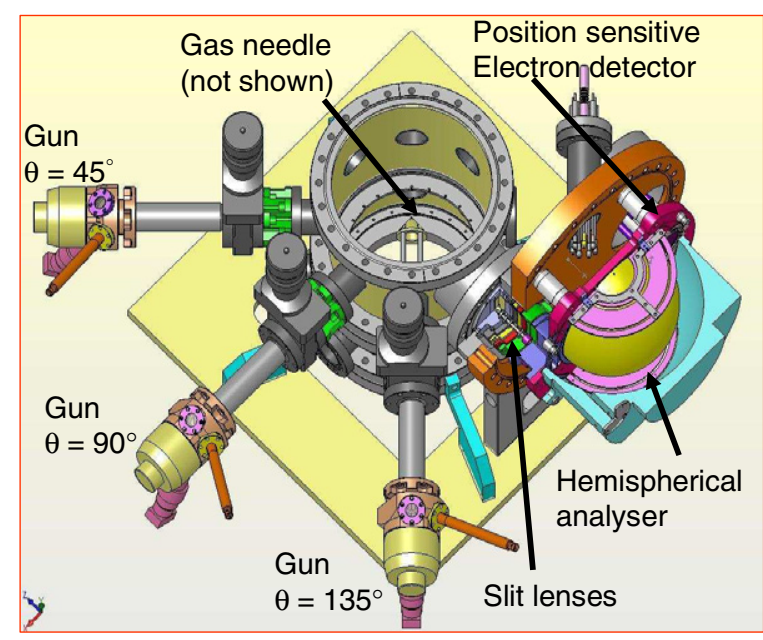

Figure 2. An overview of the spectrometer used. The scattering angle can be selected by mounting the gun on any of the ports shown. At the exit plane of the hemispherical analyser is a two-dimensional detector.

scattering angle as well. Thus a theory based on the first Born approximation is not expected to describe the experiment perfectly. However, in all cases, except the low energy (1.55 and $2 \mathrm{keV}) 135^{\circ}$ cases without absorption, the obtained ratio is within $10 \%$ of the ratio as calculated from the Rutherford formula.

\section{Spectrometer}

The spectrometer is sketched in figure 2. It consists of a $10 \mathrm{keV}$ electron gun (Kimball Physics model EGG3101), and a hemispherical analyser. The electron gun is mounted on a $2 \frac{3}{4}^{\prime \prime}$ flange, at angles of $45^{\circ}, 90^{\circ}$ or $135^{\circ}$ with respect to the analyser. The size of the beam in the interaction region is determined by an aperture just before this region with a diameter of $0.5 \mathrm{~mm}$. The analyser operates at a pass energy of $200 \mathrm{eV}$, and can float up to $-8 \mathrm{kV}$ (hence we can measure the elastic peak for an $\approx 8 \mathrm{keV}$ incoming electron beam). In front of the analyser is a five-element slit lens, used to decelerate the scattered electrons on the entrance plane of the analyser. The design of this slit lens is based on those used in solid state electron momentum spectrometers as described elsewhere (Storer et al 1994, Vos et al 2000). At the exit plane the electrons are detected by a set of channel plates, and the impact position of the electron is determined using a resistive anode as a position-sensitive detector ( $\mathrm{psd}$ ). The four corner signals of this anode are decoupled from the high voltage using $1 \mathrm{nF}$ capacitors inside the vacuum, amplified and measured using a simultaneous sample-and-hold ADC. The radial coordinate of the psd corresponds to different energies. In this way an energy window of $20 \mathrm{eV}$ can be measured simultaneously. The analyser potential is scanned slowly, and in this way the elastic peak is moved over the entire channel plate, and artefacts due to varying efficiency of the channel plates are averaged out.

The gas (methane $99.95 \%$ pure, Coregas) is admitted in the vacuum chamber either through a needle $(1 \mathrm{~mm}$ diameter) in a cross beam configuration, or through a large diameter tube, far away from the interaction region. Scans are done alternately with the gas admitted through a needle in the interaction region (signal run) and away from the interaction region (background run). The background shows a typical solid state reflection energy loss distribution (a broad distribution peaked near $20 \mathrm{eV}$ ), and is presumably due to the halo of the electron beam hitting the needle, which was placed very close to the beam in order to minimize the size of the interaction region. The background intensity is ten times smaller than the signal intensity, and we assume that the difference of the signal and background run is due to scattering from $\mathrm{CH}_{4}$ only. The chamber is pumped with a $500 \mathrm{l} \mathrm{s}^{-1}$ turbo pump, and the measurements are done at a pressure of $1-2 \times 10^{-6}$ torr. A separate run was done using $\mathrm{H}_{2}$ gas, as it can be readily identified due to its large recoil. In spite of its small cross section the elastic peak of $\mathrm{H}$ was the only one visible, thus there are no heavy impurities in the gas handling system, and the gas-jet intensity is completely removed by the subtraction procedure described before.

Before each methane run the spectrometer was tested by measuring Xe under exactly the same conditions. Good statistics in the Xe peak are easily obtained as its elastic scattering cross section is almost two orders of magnitude larger than that of $\mathrm{CH}_{4}$. After the Xe run the gas handling system was flushed with methane three times, each time pumped out by a roughing pump and subsequently by opening the leak valve completely, until the chamber pressure was within a factor of $2-3$ of the base pressure. During all this the electronics was left switched on, and none of the settings changed. Thus the $\mathrm{CH}_{4}$ run was done under virtually the same conditions as the Xe run. The only noticeable difference is that the output of the gun was larger $(50 \%)$ with methane in the chamber compared to xenon. This is attributed to a dependence of the work function (and hence the emission) of the Ta cathode on the background gas. It is conceivable that this changes the effective energy (and energy spread) of the electron beam somewhat $(0.1-0.2 \mathrm{eV})$ due to space charge effects, but no direct evidence of this was observed.

\section{Results}

In figure 3 we show the data for the largest momentum transfer studied $(|q|=38.9 \mathrm{au})$. The methane spectra shows a second elastic peak separated by $10.48 \mathrm{eV}$ (as obtained when fitting both peaks with one Gaussians each) from the main elastic peak. The calculated separation between electrons scattering from $\mathrm{C}$ and $\mathrm{H}$ is $10.36 \mathrm{eV}$, i.e. within $1 \%$ of the calculated value an error that corresponds roughly to the accuracy of the commercial high-voltage supply used. Thus we attribute the large peak to scattering from $\mathrm{C}$, and the smaller peak to scattering from $\mathrm{H}$ atoms. The width of the two peaks is very different, and a comparison with the Xe peak measured preceding the $\mathrm{CH}_{4}$ measurement shows that even the $\mathrm{C}$ peak is shifted and slightly wider than the Xe peak. The measured $\mathrm{Xe}-\mathrm{C}$ splitting is $0.97 \mathrm{eV}$, slightly larger than the calculated separation $(0.86 \mathrm{eV})$, and we consider such an agreement (deviation 5 times smaller than the experimental 


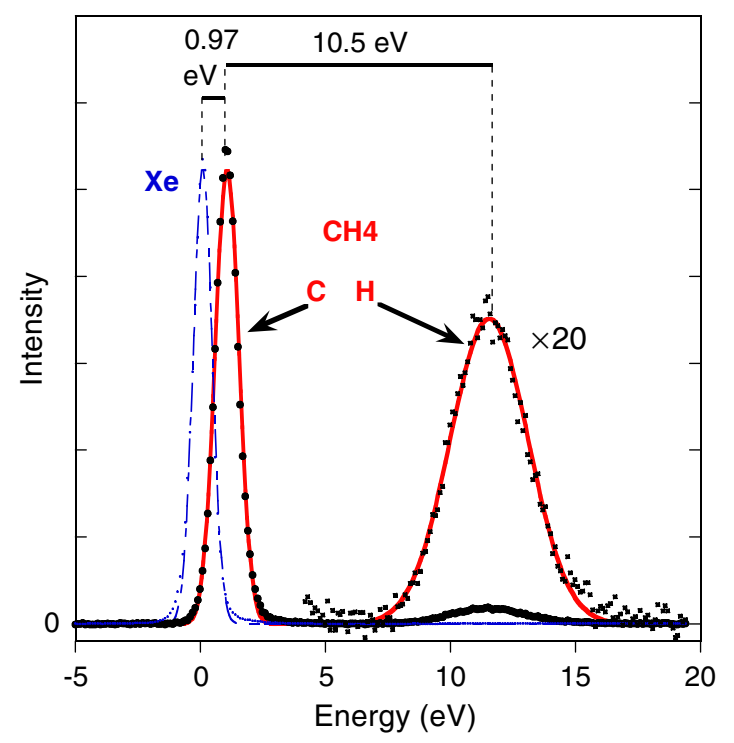

Figure 3. Spectra of methane and xenon for $6 \mathrm{keV}$ electrons scattered over $135^{\circ}$

energy resolution) as good as can be reasonably expected. For such a measurement one can determine the two peak areas, either by numerical integration over the two, completely separated, peaks, or by fitting both peaks with a Gaussian, and determining their area. As there is virtually no overlap both measurements give the same result, and the ratio of the two peak areas found ( $\mathrm{C}: \mathrm{H}$ intensity ratio of $9.1: 1)$ is in good agreement with the ratios of the Rutherford cross section (36:1) weighted by the atomic abundance (1:4). As is clear from figure 1 there is very limited difference between the Rutherford prediction and those based on the ELSEPA calculation. Thus the measured peak area ratio is within 5\% of the expected one for all the cross section models used. The possibility of the presence of a small inelastic background under the H peak will be discussed in section 5 .

At the smallest momentum transfer studied in this work (19.7 au, corresponding to the largest momentum transfer studied in Cooper et al (2007)), the analysis is much less straightforward. Now the $\mathrm{H}$ and $\mathrm{C}$ peaks are not fully separated. The $\mathrm{C}$ peak is much more intense, and hence a tail extending under the $\mathrm{H}$ peak has to be taken into account correctly, if one wants to extract a meaningful area ratio. Unfortunately, a simple Gaussian fit, although adequate for extracting the $\mathrm{C}$ intensity within $1 \%$ or so, does not describe the line shape of either $\mathrm{C}$ or Xe very well near the tail of the spectrum, where the $\mathrm{H}$ peak is. The deviation in the fit is not small, if one wants to extract the $\mathrm{H}$ area with an accuracy of $10 \%$. Fitting each peak with a single Gaussian results in a poor fit (see figure 4(a)) and the residuals in the Xe fit with a single Gaussian suggest that a tail exists extending to $2 \mathrm{eV}$ energy loss. If a similar tail exists for the $\mathrm{C}$ peak then the obtained $\mathrm{H}$ area could be too large. This interpretation is further supported by the fact that the $\mathrm{H}-\mathrm{C}$ separation of the Gaussians of the fit $(2.49 \mathrm{eV})$ is slightly less than the calculated separation $(2.66 \mathrm{eV})$ and also slightly smaller than the actual separation of the maxima of the $\mathrm{C}$ and $\mathrm{H}$ derived intensities. The $\mathrm{C}: \mathrm{H}$ intensity ratio obtained in this way is $8.7: 1$, in reasonable agreement with the Rutherford prediction (9:1), but somewhat smaller than the prediction based on ELSEPA calculations with absorption (9.9:1).

We can improve the fit markedly by fitting the $\mathrm{C}$ peak with two Gaussians, centred at slightly different energies and with different widths. The fit follows the $\mathrm{H}$ part of the spectrum more accurately, and the obtained $\mathrm{C}: \mathrm{H}$ ratio changes to $9.8: 1$, in excellent agreement with the ELSEPA calculation, including absorption (figure 4(b)). Alternatively one can start with fitting the Xe peak with a number (3 in our case) of Gaussians. If one assumes that the Xe line-shape is the spectrometer performance, then one would expect the $\mathrm{C}$ and $\mathrm{H}$ peaks in $\mathrm{CH}_{4}$ to be the spectrometer response convoluted with a Gaussian corresponding to the Doppler broadening of $\mathrm{C}$ and $\mathrm{H}$. This approach has two fewer fitting parameters for the $\mathrm{CH}_{4}$ spectrum, the fit is slightly poorer than the previous fit, and the $\mathrm{C}: \mathrm{H}$ ratio obtained in this way is $10.7: 1$. This ratio is in agreement with the ELSEPA ratio without absorption (10.7:1) (figure 4(c)). Thus a larger error has to be assigned to the extracted $\mathrm{C}: \mathrm{H}$ intensity ratio at such a low momentum transfer, and we conservatively take the intensity ratio to be $10 \pm 1: 1$. We want to stress again that these fitting ambiguities largely disappear at higher momentum transfers.

All these fitting procedures assume Gaussian line-shapes of the $\mathrm{C}$ and $\mathrm{H}$. At low momentum transfer final state effects could change the line-shape for the $\mathrm{C}$ and $\mathrm{H}$ peak, see for example the discussion in Vos and Went (2006). The importance of final state effects should decrease with increasing momentum transfer. Thus the interpretation of the data at low momentum transfer is not straightforward. All problems that affect the interpretation here (overlap of $\mathrm{C}$ and $\mathrm{H}$ peak, influence of the cross section on model chosen, influence of final state effects on line-shape) become less significant if the momentum transfer is increased. The best estimates obtained from these fittings of the various parameters are given in table 2.

In the picture sketched here the results depend only on the momentum transfer. It is thus instructive to compare the results obtained for the same momentum transfer, but at different scattering angles. This is done in figure 4(d) for the McMaster measurement employing a $100^{\circ}$ scattering angle and $2250 \mathrm{eV}$ (Cooper et al 2007), and the measurements from the Australian National University at $90^{\circ}(2640 \mathrm{eV})$ and $135^{\circ}(1550 \mathrm{eV})$ scattering angle. All measurements have a momentum transfer of $19.7 \mathrm{au}$ and the spectra are normalized such that the carbon peak has the same area. The main difference is a slightly poorer energy resolution of the McMaster experiment.

One of the consequences of equation (1) is that the width of the peaks increases proportional to the momentum transfer. In order to compare peak shapes at different energies, one can do a so-called $y$-transform, as established in the neutron Compton literature (see e.g. Watson 1996). One first subtracts the recoil energy of hydrogen from the energy loss scale. If the zero point of the energy scale was exactly known, then this should result in the $\mathrm{H}$ contribution being centred at zero energy loss. In practice the zero point is not known exactly, and one subtracts the energy corresponding to the maximum of the fit of the $\mathrm{H}$ component. Next one 

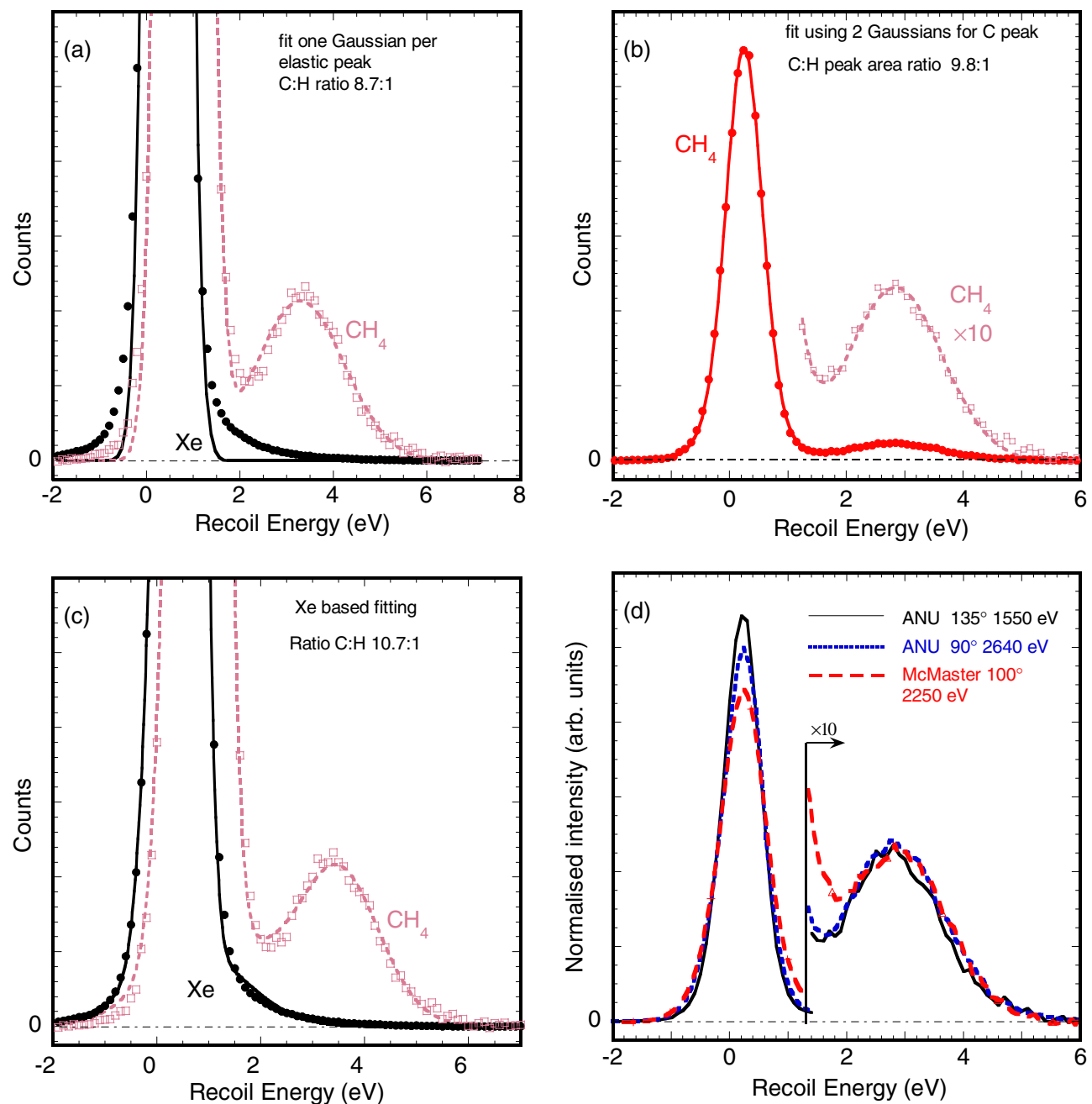

Figure 4. Panels (a)-(c) show the effect of the analysis method on the ratios obtained for the low momentum transfer measurement $\left(1.55 \mathrm{keV}\right.$ and $135^{\circ}$ scattering angle), as explained in the main text. Panel (d) shows a comparison of three measurements at the same momentum transfer (19.7 au) two at different scattering angles at the Australian National University, and the previously published measurement from McMaster University (Cooper et al 2007). This plot illustrates that the resolution in the current work is slightly better than the earlier McMaster experiment.

Table 2. A summary of the experimental results for different energies $E_{0}$ and scattering angles. The measured C-Xe peak separation is compared with the calculated one, the peak widths $(\sigma)$ for $\mathrm{Xe}, \mathrm{C}$ and $\mathrm{H}$ are given as well. The width of the $\mathrm{H}$ peak is also given after transformation of the energy scale into a momentum scale, as explained in the text. The last column displays the intensity ratio of the $\mathrm{C}$ and $\mathrm{H}$ peak. Data in the lower row are from the McMaster group (Cooper et al 2007)

\begin{tabular}{|c|c|c|c|c|c|c|c|c|c|c|c|}
\hline \multirow[b]{2}{*}{$\begin{array}{l}E_{0} \\
(\mathrm{keV})\end{array}$} & \multirow[b]{2}{*}{$\begin{array}{l}\theta \\
\left({ }^{\circ}\right)\end{array}$} & \multirow[b]{2}{*}{$\begin{array}{l}q \\
(\mathrm{au})\end{array}$} & \multicolumn{2}{|c|}{ Xe-C sep. } & \multicolumn{2}{|c|}{ C-H sep. } & \multirow{2}{*}{$\begin{array}{l}\sigma \mathrm{Xe} \\
\text { Obs. } \\
(\mathrm{eV})\end{array}$} & \multirow{2}{*}{$\begin{array}{l}\sigma \mathrm{C} \\
\text { Obs. } \\
(\mathrm{eV})\end{array}$} & \multicolumn{2}{|c|}{$\sigma \mathrm{H}$} & \multirow{2}{*}{$\begin{array}{l}\mathrm{I}_{C}: \mathrm{I}_{H} \\
\text { Obs. }\end{array}$} \\
\hline & & & $\begin{array}{l}\text { Calc. } \\
(\mathrm{eV})\end{array}$ & $\begin{array}{l}\text { Obs. } \\
\text { (eV.) }\end{array}$ & $\begin{array}{l}\text { Calc. } \\
\text { (eV) }\end{array}$ & $\begin{array}{l}\text { Obs. } \\
(\mathrm{eV})\end{array}$ & & & $\begin{array}{l}\text { Obs. } \\
(\mathrm{eV})\end{array}$ & $\begin{array}{l}\text { Obs. } \\
(\mathrm{au})\end{array}$ & \\
\hline 2.65 & 90 & 19.7 & 0.22 & 0.36 & 2.67 & 2.64 & 0.32 & 0.39 & 0.82 & 2.58 & 9.1 \\
\hline 4.00 & 90 & 24.3 & 0.33 & 0.53 & 4.04 & 4.04 & 0.34 & 0.42 & 0.99 & 2.55 & 9.1 \\
\hline 6.00 & 90 & 29.8 & 0.50 & 0.79 & 6.07 & 6.13 & 0.35 & 0.40 & 1.21 & 2.64 & 9.1 \\
\hline 8.00 & 90 & 34.4 & 0.67 & 0.70 & 8.11 & 8.2 & 0.42 & 0.50 & 1.37 & 2.57 & 9.4 \\
\hline 1.55 & 135 & 19.7 & 0.22 & 0.35 & 2.66 & 2.67 & 0.29 & 0.34 & 0.79 & 2.53 & 10 \\
\hline 2.00 & 135 & 22.4 & 0.28 & 0.40 & 3.44 & 3.37 & 0.30 & 0.35 & 0.968 & 2.77 & 9.4 \\
\hline 4.00 & 135 & 31.7 & 0.57 & 0.71 & 6.89 & 6.92 & 0.35 & 0.43 & 1.27 & 2.60 & 9.4 \\
\hline 6.00 & 135 & 38.9 & 0.86 & 0.97 & 10.36 & 10.48 & 0.38 & 0.47 & 1.55 & 2.62 & 9.1 \\
\hline 2.25 & 100 & 19.7 & 0.22 & - & 2.66 & 2.66 & - & - & 0.89 & & 9.2 \\
\hline
\end{tabular}

divides the energy scale (in Hartree) by $q / M$. (in atomic units the mass of $\mathrm{H}$ is $\approx 1836$ ). The results are shown in figure 5. The distribution obtained in this way should correspond to the Compton profile of the momentum distribution (in atomic unit of momentum) for $\mathrm{H}$ in $\mathrm{CH}_{4}$, i.e. the probability that the momentum component of $\mathrm{H}$ along the momentum transfer direction has a certain value. Indeed the curves of the low and high energy measurements after 


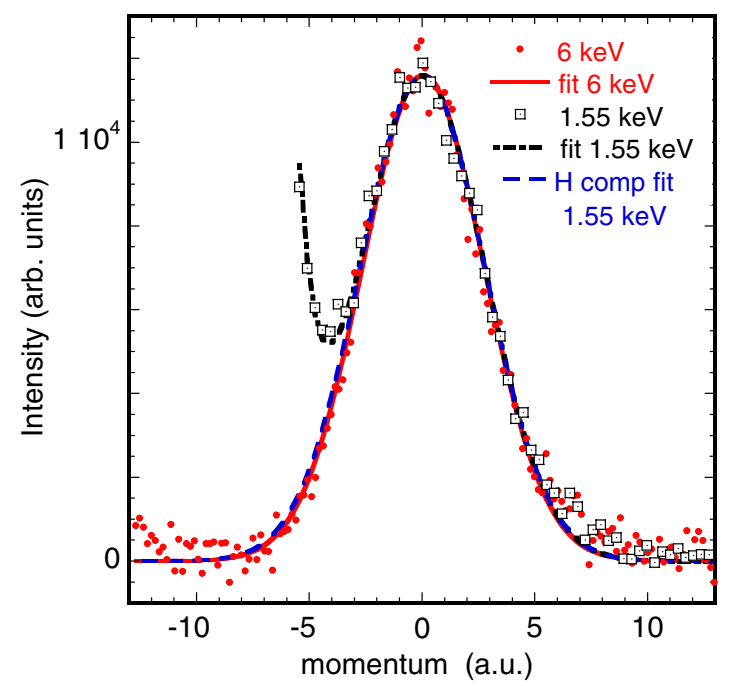

Figure 5. The hydrogen part of the elastic peak after conversion of the energy scale to a momentum scale, as explained in the main text. The scattering angle was $135^{\circ}$ for both measurements.

the $y$-transform are virtual identical, for the part of the spectra where the carbon contribution to the signal is negligibly small. This is another indication that the simple picture, sketched here, provides quite a good description of the experiment. The resulting full width half maximum for the hydrogen peak in momentum space, obtained in this way, is reproduced in table 2 as well. For this calculation we used the Xe width as an estimate of the energy resolution, and subtracted this in quadrature from the width of the hydrogen peak, as derived from the fit. The width in units of momentum is then obtained by multiplying this energy width by $\frac{m}{q}$. The resulting width $(\sigma)$ of $\approx 2.6$ au is in good agreement with considerations based on the zero-point energy of methane, as explained by Cooper et al (2007).

\section{Discussion and conclusion}

These measurements show very clearly that keV electrons scattering elastically over large angles transfer energy to the scattering atoms and this energy can be calculated by assuming that the electron scatters from a free atom, even if this atom is part of a larger, much heavier molecule. This is even the case if the energy transferred to the atom is much smaller than the energy of the chemical bond. These observations can be understood in terms of the plane-wave-impulse approximation developed in neutron Compton scattering literature (Watson 1996). In these neutron experiments similar amounts of momentum are transferred to the nucleus and here one also describes the final state of the nucleus as a plane wave (i.e. as a free particle). The key to the understanding of this phenomenon is the time scale of the collision. In oversimplified terms, the collision is over before the scattering atom can move, and hence feel the potential well that keeps the atom bound to the molecule. After the collision the kinetic energy of the scattering atom will be transferred to vibrational and rotational excitations of the molecule, a change in its centre-of-mass kinetic energy and possibly electronic excitations. The process is 'elastic' in the sense that during the collision the process can be described as a collision between free particles (total kinetic energy is conserved). Sometimes these collisions are referred to as 'quasi-elastic' (see e.g. Cooper et al 2007).

The energy separation found between $\mathrm{H}$ and $\mathrm{C}$ in methane is in agreement with that expected for scattering from free $\mathrm{H}$ and $\mathrm{C}$. This agreement is as good as can be expected considering our finite energy resolution and the intrinsic width of these peaks. The average difference between experimental and calculated separation is of the order of $0.05 \mathrm{eV}$, and the deviations seem to be of a statistical nature. We take $0.05 \mathrm{eV}$ as the accuracy which we can determine the $\mathrm{H}-\mathrm{C}$ separation.

The separation found between $\mathrm{Xe}$ and $\mathrm{C}$ peaks increases with increasing momentum transfer, and hence it certainly contains a signature of the recoil energy transferred to $\mathrm{C}$ atoms. However the separation seems systematically somewhat larger than what is calculated (by about $0.15 \mathrm{eV}$ ) for scattering from free $\mathrm{Xe}$ and $\mathrm{C}$ particles. We think that this is a consequence of space charge effects or surface potential changes, as the output of the gun generally increases by $50 \%$ if the Xe gas is replaced by $\mathrm{CH}_{4}$. This makes us also somewhat hesitant to interpret quantitatively the difference in width of $\mathrm{Xe}$ and $\mathrm{C}$ peak in terms of Doppler broadening, as part of this could be due to a change in energy spread of the electron beam. These problems could probably be circumvented by differentially pumping the gun, hence not exposing the filament to the $\mathrm{Xe}$ and $\mathrm{CH}_{4}$ background gas.

The ratio of the $\mathrm{C}$ and $\mathrm{H}$ peak is, especially at high momentum transfer, in good agreement with the expected intensity ratio. At high momentum transfer the difference in differential cross section calculated using various models (Rutherford, potential scattering, potential scattering including absorption) is small (5\%), and indeed the experiment is close to these values. The accuracy of the experimental peak area ratio is estimated to be better than $5 \%$ at high momentum transfer, and $10 \%$ at the lower momentum transfer values. It is thus hard to determine experimentally which model is best. Also the atomic approach for the cross section calculation (i.e. the assumption that the charge distribution around the nucleus in a molecule is the same as the charge distribution around this molecule in an isolated atom) used here may not be fully applicable to a molecule.

In the previous analysis we have assumed that all counts in the spectra are either due to electrons scattered elastically from $\mathrm{H}$ or $\mathrm{C}$. This is a slight oversimplification. To illustrate this point we show in figure 6 a spectra taken at $90^{\circ}$ scattering angle and an energy of $1550 \mathrm{eV}$. Under these rather low momentum transfer conditions, the signal of electrons scattering from $\mathrm{H}$ is not completely separated but is only apparent as a shoulder. We did not try to analyse these spectra quantitatively in terms of $\mathrm{C}: \mathrm{H}$ peak ratio due to the ambiguity of the fit.

We plot in figure 6 the result of the signal run (gas through needle) and the background run (gas admitted in vacuum chamber away from electron beam, see section 3). In the background run we see a peak due to electrons scattering from the background gas and from the needle as well as dark count rate of the channel plates. After subtracting the background 

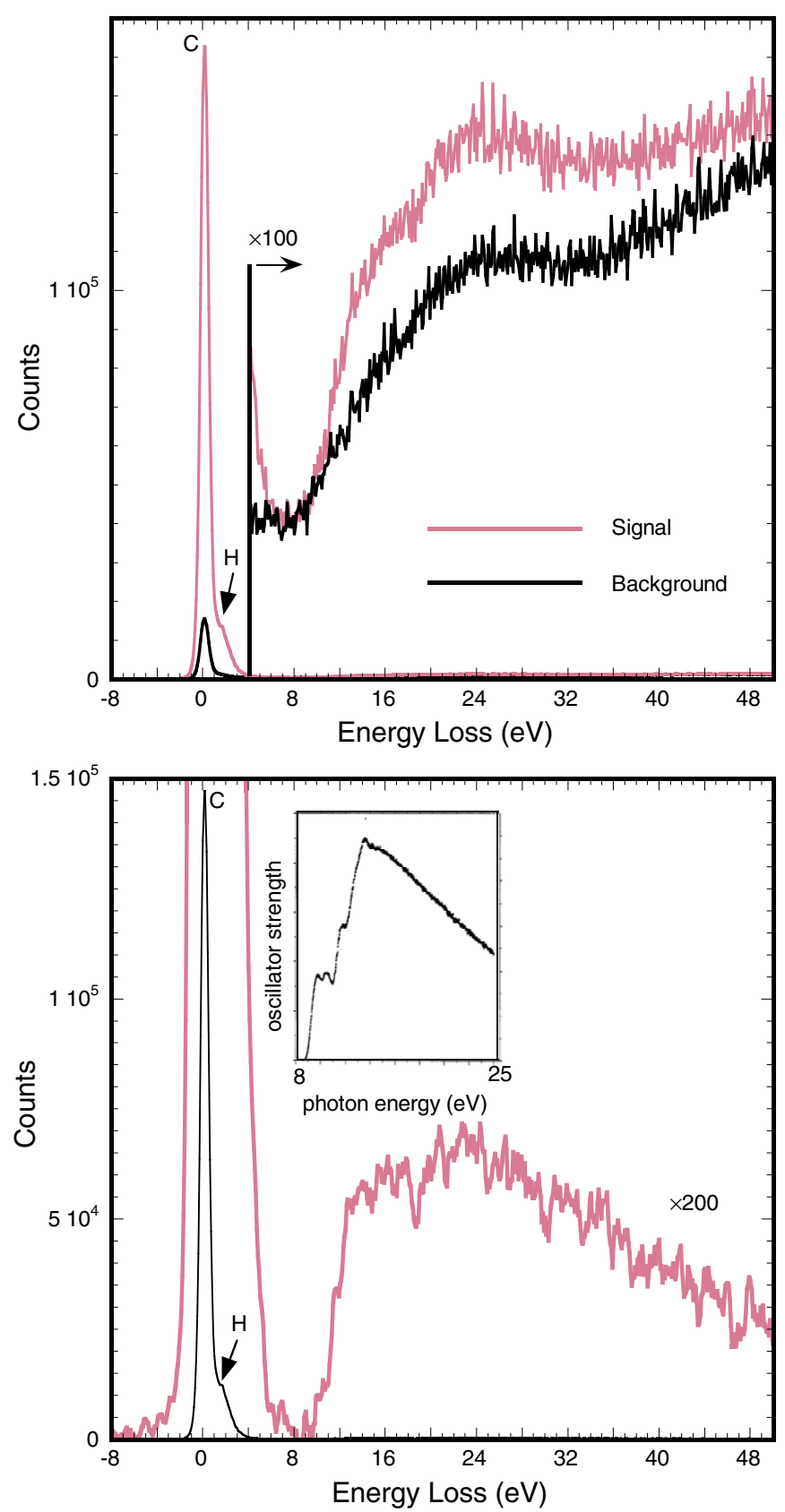

Figure 6. The signal and background intensity of $1550 \mathrm{eV}$ electrons scattered over $90^{\circ}$ from methane is shown in the top panel. The lower panel shows the background subtracted signal intensity which displays, besides the elastic peak, a small but non-zero intensity at energy loss values over $8 \mathrm{eV}$. The inset shows a generalized oscillator strength measurement by Au et al (1993) which shows a similar onset.

run from the signal run we get the results of methane only. This is plotted in the lower part of figure 6. As mentioned before, $\mathrm{H}$ shows up as a shoulder on the carbon peak. At about $7 \mathrm{eV}$ at either side of the elastic peak the backgroundsubtracted intensity is, within the statistical accuracy, equal to zero. However around $9-10 \mathrm{eV}$ a very small intensity reappears, which persists up to very large energy loss values. The intensity is very weak, requiring long measurement times. (a week or so, even under these relatively low momentum transfer conditions).
A possible interpretation is that this is due to electrons that have scattered elastically from $\mathrm{CH}_{4}$ and left the scatterer in an excited electronic state. This process is generally referred to as absorption in the elastic scattering literature. This can be supported by e.g. generalized oscillator strength measurements, which also show a delay in the onset of the intensity, which is the result of the fact that a minimum energy is required to excite methane into an electronically excited state (see e.g. Au et al 1993). The background was measured from 6 to $90 \mathrm{eV}$ and the total area of this background was $8.5 \%$ of the area of the total elastic peak. Absorption effects are expected to influence the differential elastic scattering cross section by this order of magnitude for scattering at these energies from small atoms/molecules (Salvat 2003). There are two ways that this process can influence the results presented in this paper.

- Absorption could affect electrons scattered from $\mathrm{C}$ in a different way from electrons scattered from $\mathrm{H}$. If the $8.5 \%$ reduction is not evenly divided over both elements, then it could change the observed $\mathrm{C}: \mathrm{H}$ intensity ratio.

- At the higher momentum transfer values the H peak would overlap with the inelastic background due to electrons scattering from $\mathrm{C}$ and leaving the target in an excited state. This background, if not included in the fit, would cause an apparent increase in the $\mathrm{H}$ peak intensity. The size of the background can be judged at larger energy loss values (see e.g. figure 3), but the background was not obvious with the statistics obtainable at higher momentum transfer.

From measurements on Xe where these effects are much easier to measure mainly due to the much larger elastic cross section, we know that the observed intensity of the background (relative to the elastic peak itself) is roughly inversely proportional to the energy of the incoming beam. Thus we do not think that these effects change the $\mathrm{C}$ to $\mathrm{H}$ peak intensity ratio by more than $10 \%$ for the $1550 \mathrm{eV}$ experiment and less than $5 \%$ for the $6 \mathrm{keV}$ experiment.

We have presented here a series of measurements that covers the whole range of momentum transfers used in the electron scattering experiment from polyethylene and formvar. The results and the current results are summarized in figure 7. At the lower end of the studied range of momentum transfers the $\mathrm{C}: \mathrm{H}$ ratio obtained depends somewhat on the analysis method, and theoretical model used. At higher momentum transfers all these problems vanish, and the obtained peak intensity ratio agrees well with theory and certainly no 'anomalies' of the order of 20-30\% are observed, as is the case for neutron and electron scattering experiments from polymer films.

The methane results described here could be interpreted as a sign that the 'anomalous' scattering results are specific to condensed matter. However the methane results contrast strongly with the recently reported difference in the spectra of high-energy electrons deflected over large angles for 50\% $\mathrm{H}_{2}-\mathrm{D}_{2}$ mixtures and $\mathrm{HD}$ (Cooper et al 2008). In that work it was concluded that the $\mathrm{H}$ and $\mathrm{D}$ derived peak areas are the same in $\mathrm{HD}$, but in the $50 \% \mathrm{H}_{2}-\mathrm{D}_{2}$ mixture the $\mathrm{H}$ derived signal is $30 \%$ weaker than the $\mathrm{D}$ signal. 


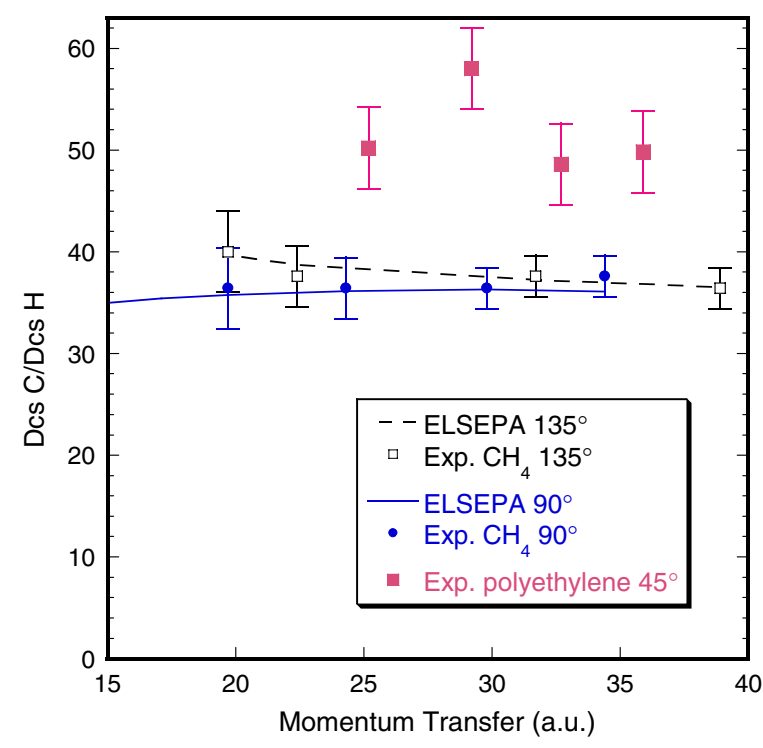

Figure 7. The experimental ratio of the differential elastic scattering cross section of $\mathrm{C}$ and $\mathrm{H}$ as calculated using the ELSEPA package (including absorption) and the experimentally obtained values. Also shown the C:H cross section ratio obtained for solid polyethylene as was described by Vos et al (2004).

In conclusion we have presented elastic scattering results from methane at high momentum transfer. Clear separation was found between the signal of electrons scattered from either $\mathrm{C}$ and $\mathrm{H}$ atoms. The elastic peak of electrons scattered from $\mathrm{H}$ shows a clear Compton profile of the proton momentum distribution. The intensity ratio of the $\mathrm{C}$ and $\mathrm{H}$ peak was within experimental error in agreement with the expectation based on cross section calculations. The latter observation is in strong contrast to scattering experiments at similar momentum transfer from polyethylene and formvar films (Chatzidimitriou-Dreismann et al 2003, Vos et al 2004) and gas-phase experiments using hydrogen isotopes (Cooper et al 2008).

\section{Acknowledgments}

Construction of the spectrometer was possible due to highquality technical support of the Research School and the Atomic and Molecular Physics Laboratories. In particular we are indebted to $\mathrm{Mr} \mathrm{S}$ Battisson and $\mathrm{Mr} \mathrm{S}$ Huynh. We want to thank Professor E Weigold and Professor A Hitchcock for valuable discussions and critically reading of the manuscript. MV, MRW and CAC-D acknowledges support by a Discovery Grant of the Australian Research Council. CAC-D acknowledges partial support by a grant from the Royal Swedish Academy of Sciences.

\section{References}

Abdul-Redah T and Chatzidimitriou-Dreismann C A 2002 J. Alloys Comp. 356-357 249

Au J W, Cooper G, Burton G R, Olney T N and Brion C E 1993 Chem. Phys. 173 209-39

Boersch H, Wolter R and Schoenebeck H 1967 Z. Phys. 199 124-34

Chatzidimitriou-Dreismann C A 2005 Laser Phys. 15780

Chatzidimitriou-Dreismann C A, Abdul-Redah T, Streffer R M F and Mayers J 1997 Phys. Rev. Lett. 792839

Chatzidimitriou-Dreismann C A and Stenholm S 2007 arXiv:quant-ph/0702038

Chatzidimitriou-Dreismann C A, Vos M, Kleiner C and Abdul-Redah T 2003 Phys. Rev. Lett. 9157403

Cooper G, Christensen E and Hitchcock A P 2007 J. Chem. Phys. 127084315

Cooper G, Hitchcock A P and Chatzidimitriou-Dreismann C A 2008 Phys. Rev. Lett. 100043204

Cooper G, Hitchcock A, Chatzidimitriou-Dreismann C and Vos M 2007 J. Electron Spectrosc. Relat. Phenom. 15528

Cooper M J, Mijnarends P E, Shiotani N, Sakai N and Bansil A 2004 $X$-Ray Compton Scattering (Oxford: Oxford University Press)

Cowley R A 2003 J. Phys.: Condens. Matter 15 4143-52

Fielding A L, Timms D N and Mayers J 2000 Physica B 276 69-70

Gidopoulos N I 2005 Phys. Rev. B 71 054106-5

Hohenberg P C and Platzman P M 1966 Phys. Rev. 152198

Karlsson E B 2003 Phys. Rev. Lett. 90095301

Karlsson E B and Lovesey S W 2000 Phys. Rev. A 6162714

Reiter G F and Platzman P M 2005 Phys. Rev. B 71054107

Salvat F 2003 Phys. Rev. A 6812708

Salvat F, Jablonski A and Powell C J 2005 Comput. Phys. Commun. 165 157-90

Storer P, Caprari R S, Clark S A C, Vos M and Weigold E 1994 Rev. Sci. Intrum. $\mathbf{6 5} 2214$

Varga D, Tokési K, Berényi Z, Tóth J and Kövér L 2006 Surf. Interface Anal. 38 544-7

Varga D, Tökési K, Berényi Z, Tóth J, Körvér L, Gergely G and Sulyok A 2001 Surf. Interface Anal. 31 1019-26

Vos M 2002 Phys. Rev. A 6512703

Vos M, Chatzidimitriou-Dreismann C A, Abdul-Redah T and Mayers J 2004 Nucl. Instrum. Methods B 227 233-50

Vos M, Cornish G P and Weigold E 2000 Rev. Sci. Intrum. $713831-40$

Vos M and Went M R 2006 Phys. Rev. B 74205407

Watson G I 1996 J. Phys.: Condens. Matter 85955

Went M R and Vos M, unpublished

Werner W S M, Tomastik C, Cabela T, Richter G and Störi H 2001 J. Electron Spectrosc. Relat. Phenom. 113 127-35

Yubero F, Rico V J, Espinos J P, Cotrino J and Gonzalez-Elipe A R 2005 Appl. Phys. Lett. 87084101 